Check for updates

Cite this: Phys. Chem. Chem. Phys., 2020, 22, 21185

Received 29th June 2020, Accepted 4th September 2020

DOI: 10.1039/d0cp03473h

rsc.li/pccp

\title{
Heterogeneous dynamics in partially disordered proteins $\dagger$
}

\author{
Salla I. Virtanen, $\$$ Anne M. Kiirikki, $\$$ Kornelia M. Mikula, Hideo Iwaï (DD and \\ O. H. Samuli Ollila $1 D$ *
}

\begin{abstract}
Importance of disordered protein regions is increasingly recognized in biology, but their characterization remains challenging due to the lack of suitable experimental and theoretical methods. NMR experiments can detect multiple timescale dynamics and structural details of disordered protein regions, but their detailed interpretation is often difficult. Here we combine protein backbone ${ }^{15} \mathrm{~N}$ spin relaxation data with molecular dynamics (MD) simulations to detect not only heterogeneous dynamics of large partially disordered proteins but also their conformational ensembles. We observed that the rotational dynamics of folded regions in partially disordered proteins is dominated by similar rigid body rotation as in globular proteins, thereby being largely independent of flexible disordered linkers. Disordered regions, on the other hand, exhibit complex rotational motions with multiple timescales below $\sim 30$ ns which are difficult to detect from experimental data alone, but can be captured by MD simulations. Combining MD simulations and backbone ${ }^{15} \mathrm{~N}$ spin relaxation data, measured applying segmental isotopic labeling with salt-inducible split intein, we resolved the conformational ensemble and dynamics of partially disordered periplasmic domain of TonB protein from Helicobacter pylori containing 250 residues. To demonstrate the universality of our approach, it was applied also to the partially disordered region of chicken Engrailed 2. Our results pave the way in understanding how TonB transfers energy from inner membrane to the outer membrane receptors in Gram-negative bacteria, as well as the function of other proteins with disordered domains.
\end{abstract}

\section{Introduction}

Long ( $>30$ residue) disordered segments that lack the well defined 3D structure are found in $33 \%$ of eukaryotic proteins. ${ }^{1}$ These intrinsically disordered protein regions are associated with various diseases, including cancer, cardiovascular diseases, neurodegenerative diseases, and diabetes, ${ }^{2}$ yet their importance in biology is probably underestimated because they are difficult to characterize with the available experimental and theoretical methods. Intrinsically disordered proteins and regions participate in important functions also in bacteria, archaea and viruses. ${ }^{3}$ Furthermore, the importance of disordered linkers attached to folded protein domains is increasingly recognized in allosteric regulation, complex formation, biocatalysis and protein design. ${ }^{4-8}$ To fully understand the role of disordered protein regions in biology and exploit them in protein design, robust tools to determine their conformational ensembles and dynamics are needed. Average properties and distances between individual

Institute of Biotechnology, University of Helsinki, Helsinki, Finland.

E-mail: samuli.ollila@helsinki.fi

$\dagger$ Electronic supplementary information (ESI) available. See DOI: 10.1039/d0cp03473h

\$ These authors contributed equally to this work. residues of disordered proteins can be accessed using small angle X-ray scattering (SAXS), electron paramagnetic resonance (EPR) or fluorescence experiments. ${ }^{9,10}$ On the other hand, nuclear magnetic resonance (NMR) experiments can detect multiple timescale dynamics and structural details at atomic resolution in disordered and partially disordered proteins. However, NMR experiments are limited by the spectral overlap and complications in the interpretation of the data, especially for large and partially disordered proteins. ${ }^{411-14}$

We have recently resolved the problem of spectral overlap for partially disordered TonB protein with 101 folded and 149 disordered residues using segmental isotopic labeling with salt-inducible split intein. ${ }^{16}$ Moreover, we have used molecular dynamics (MD) simulations to interpret the rotational dynamics of asymmetrically shaped folded proteins from NMR spin relaxation times. ${ }^{15}$ However, the accuracy of force field parameters limits the applications of MD simulations to interpret the NMR data. For folded proteins, the main issue has been the underestimated water viscosity in the commonly used TIP3P water model, which leads to too fast rotational diffusion of rigid proteins. ${ }^{15,17}$ Nevertheless, this can be taken into account when calculating NMR parameters even for proteins with anisotropic shape. ${ }^{15}$ For disordered proteins, canonical force fields predict too 
condensed conformational ensembles and calculated NMR parameters often disagree with experiments. ${ }^{18-22}$ Attempts to overcome these issues have been made by reweighting the conformational ensembles $^{23}$ or rescaling the timescales to reproduce the experimentally measured NMR spin relaxation times. ${ }^{22,24}$ While these approaches can be useful in the interpretation of individual experiments, they cannot be generally applied for proteins with heterogeneous dynamics in complex environments, such as membrane bound disordered linkers. Furthermore, rescaling of timescales is ambiguous for disordered proteins without clearly distinguishable dynamical processes, and ensemble reweighting can be used only when the original ensemble is not too far from the correct solution.

Here, we show that NMR spin relaxation times and MD simulations can be combined to resolve conformational ensembles of proteins with heterogeneous dynamics such as partially disordered proteins. We resolve conformational ensembles of two large ( $>100$ residues) partially disordered proteins: periplasmic domain (residues 36-285) of TonB protein from Helicobacter pylori (HpTonB) and residues 143-259 of chicken Engrailed 2 (EN2). Periplasmic domain of TonB propagates energy created by proton motive force in the inner membranes of Gram-negative bacteria to the TonB dependent receptors (TBDT) in the outer membranes via unknown mechanism. ${ }^{25}$ Engrailed 2 is a transcription factor in which partially disordered residues 143-259 are highly conserved and crucial, in particular, in the binding of transcriptional regulators. ${ }^{26-28}$ The resolved conformational ensembles give novel insight in the rotational timescales of disordered protein regions and in the biological function of TonB proteins. Furthermore, our results pave the way in understanding of relevant properties and functions of disordered protein regions in biology and protein engineering.

\section{Results and discussion}

\section{Resolving conformational ensembles of partially disordered proteins using spin relaxation data and MD simulations}

The periplasmic domain of TonB protein in Gram-negative bacteria consists of the folded C-terminal region (residues 185-285 in HрTonB) and disordered proline rich region (residues 31-184 in HpTonB, shown in Fig. 1A). ${ }^{16}$ We have recently determined the NMR structure of the folded C-terminal domain in HpTonB from a construct containing the last 107 residues $\left(H_{p}\right.$ TonB $\left._{179-285}\right)$ and measured the backbone $\mathrm{N}-\mathrm{H}$ spin relaxation times of almost all residues in the periplasmic region of TonB protein from Helicobacter pylori (HpTonB $\left.{ }_{36-285}\right)$ using the segmental isotopic labeling with salt-inducible split intein. ${ }^{16,29}$ The main conclusion from these experiments is that the structure and dynamics of the folded C-terminal domain is largely independent of the proline rich disordered region. However, more detailed interpretation of the conformational ensemble and dynamics of $H p$ TonB $_{36-285}$ containing disordered region cannot be made from the experimental data alone.

To resolve the conformational ensemble of partially disordered periplasmic domain of $H p$ TonB, we initiated MD simulations of

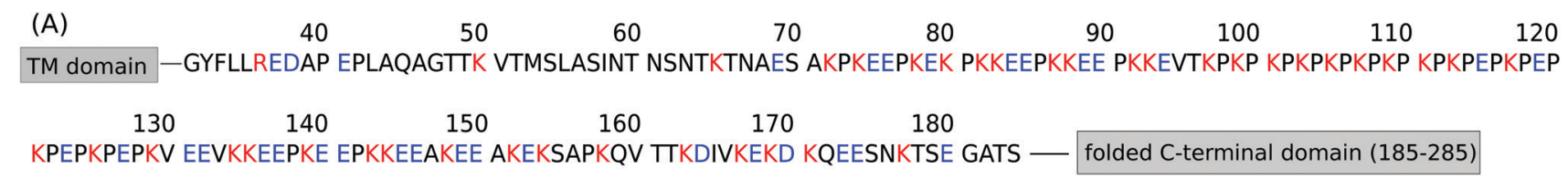

(B)

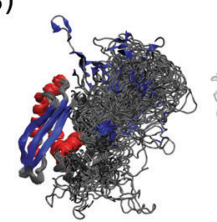

Amber ff99SB-ILDN

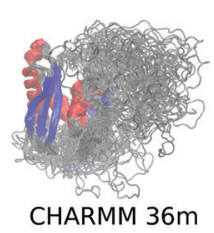

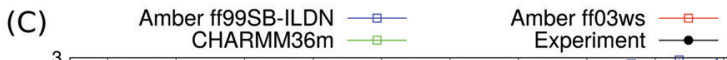

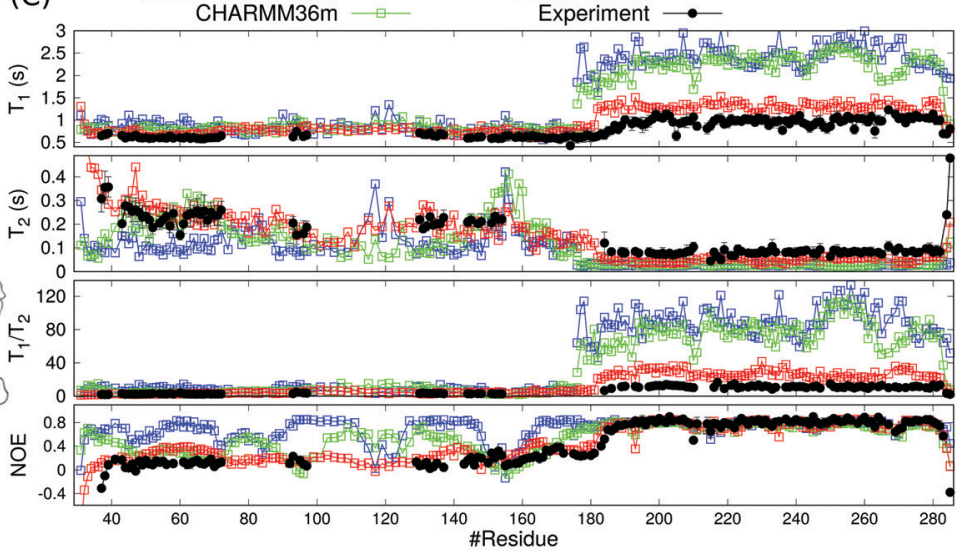

Fig. 1 (A) Sequence of the disordered proline rich region (residues 31-184) in HpTonB. The trans membrane (TM) domain is anchored in the inner membrane of Gram-negative bacteria and the folded C-terminal domain interacts with TonB dependent receptors in the outer membrane. Aminoacids

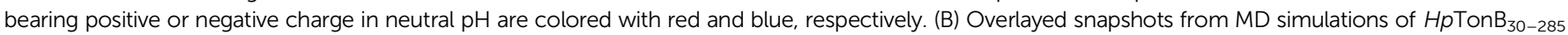
with different force fields without additional salt. The orientation of folded domain is fitted in each snapshot. Residues within $\alpha$-helices and $\beta$-strands are red and blue, respectively. (C) Spin relaxation times calculated from simulations with different force fields compared with the experimental values without additional salt. Rotational diffusion of the folded regions in Amber ff99SB-ILDN and CHARMM36m simulations is divided by a factor 2.9 in the spin relaxation time calculation to correct underestimated viscosity of TIP3P water model. ${ }^{15}$ 
TonB $_{30-285}$ starting from the fully extended linker conformation using three different force fields. In simulations with the Amber ff99SB-ILDN ${ }^{30}$ and CHARMM $36 \mathrm{~m}^{31}$ force fields, the linker rapidly collapsed (40 ns and $120 \mathrm{~ns}$, respectively) and the protein formed compact structures with the radius of gyration of $2.29 \pm 0.02 \mathrm{~nm}$ and $2.32 \pm 0.05 \mathrm{~nm}$, respectively (Fig. 1B). The Amber ff03ws force field $^{32}$ resulted in significantly more extended structure with the radius of gyration of $3.5 \pm 0.2 \mathrm{~nm}$. To evaluate the results from different force fields against experimental data, we calculated the backbone ${ }^{15} \mathrm{~N}$ spin relaxation times, $T_{1}, T_{2}$ and NOE relaxation, from simulations taking into account the effect of incorrect water viscosity in TIP3P water model for the folded region (Fig. 1C). ${ }^{15}$ Amber ff99SB-ILDN and CHARMM36m force fields with the collapsed structures overestimate the $T_{1} / T_{2}$ ratios for the C-terminal domain, suggesting that the rotation of the folded region is too slow in these simulations. In Amber ff03ws simulations with more extended conformational ensemble, the rotation of C-terminal domain is faster and the spin relaxation times are in better agreement with experiments. Therefore, we conclude that the simulations with Amber ff99SB-ILDN and CHARMM36m force fields predict overcondensated conformations for the disordered proline rich region, and that the whole protein rotates as a single relatively rigid body in these simulations with significantly slower timescales than the C-terminal domain without the disordered region attached. In more realistic Amber ff03ws simulations, the disordered region with more extended conformations has only a marginal effect on the rotational dynamics of C-terminal domain, in line with the interpretation from experimental spin relaxation data. ${ }^{16}$
Overcondensation of disordered proteins in Amber ff99SB-ILDN and CHARMM36m simulations is reported also previously. ${ }^{19,20}$

Even though the spin relaxation times from the Amber ffo3ws simulation are close to the experimental values in Fig. 1C, they slightly overestimate the $T_{1} / T_{2}$ ratios for residues in the C-terminal domain suggesting that the rotational dynamics of the folded region is still too slow when compared with experiments. To simulate the protein in conditions closer to experiments, we added $40 \mathrm{mM} \mathrm{NaCl}$ to the system, which approximately corresponds to the ionic strength of $20 \mathrm{mM}$ sodium phosphate buffer solution at $\mathrm{pH} 6$ that was used in the experiments. Indeed, the addition of salt led to the further extension of the disordered linker and faster rotational diffusion of the structured C-terminal domain (Table 1). Spin relaxation times from the simulation with $40 \mathrm{mM}$ of $\mathrm{NaCl}$ are in good agreement with the experimental values (Fig. 2). Further increase of $\mathrm{NaCl}$ concentration to $150 \mathrm{mM}$ did not affect the results neither in experiments nor in simulations (Fig. 2). The radius of gyration from simulations with $150 \mathrm{mM}$ $\mathrm{NaCl}(5.3 \pm 0.7 \mathrm{~nm})$ was close to the experimental value $(5.5 \pm 0.7 \mathrm{~nm})$ measured with the same salt concentration. In conclusion, both spin relaxation times and radius of gyration from the Amber ff03ws simulation agree with experiments when electrostatic conditions of solvent match with experiments, suggesting that this simulation can be used to interpret the spin relaxation times of partially disordered $H p$ TonB $_{36-285}$ protein.

Extension of the disordered linker region upon the increase of $\mathrm{NaCl}$ concentration from 0 to $40 \mathrm{mM}$ can be explained by the

Table 1 Rotational diffusion coefficients $\left(\mathrm{rad}^{2} \cdot 10^{7} \mathrm{~s}^{-1}\right.$ ) of folded C-terminal domain (residues 196-280) of HpTonB $30-285$ and radiuses of gyrations of $\mathrm{HpTonB}_{30-285}$ with different concentrations of additional $\mathrm{NaCl}$ from simulations with Amber ffO3ws force field

\begin{tabular}{llllll}
\hline & $D_{x}$ & $D_{y}$ & $D_{z}$ & $D_{\mathrm{av}}$ & $\tau_{\mathrm{c}}$ \\
\hline $0 \mathrm{mM}$ & $1.3 \pm 0.1$ & $1.3 \pm 0.1$ & $1.4 \pm 0.2$ & $1.3 \pm 0.2$ & $13 \pm 2$ \\
$40 \mathrm{mM}$ & $1.80 \pm 0.01$ & $2.1 \pm 0.2$ & $2.4 \pm 0.3$ & $2.1 \pm 0.2$ & $7.9 \pm 0.8$ \\
$150 \mathrm{mM}$ & $1.65 \pm 0.03$ & $1.7 \pm 0.1$ & $4.1 \pm 0.3$ & $2.5 \pm 0.2$ & $6.6 \pm 0.6$
\end{tabular}
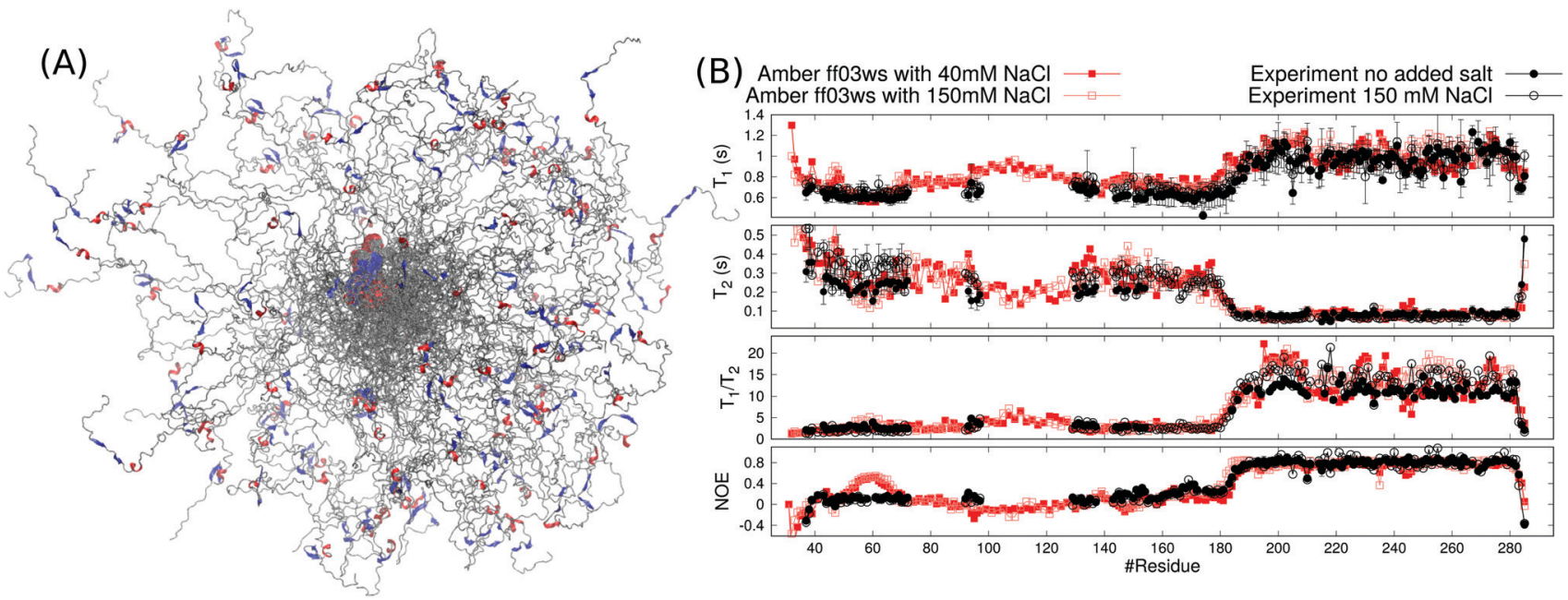

Fig. 2 (A) Overlayed snapshots from Amber ff03ws MD simulations of $H p T o n B_{30-285}$ with $40 \mathrm{mM} \mathrm{NaCl}$. The orientation of folded domain is fitted in each snapshot and color codes for secondary structure are similar to Fig. 1B. (B) Spin relaxation times calculated from Amber ff03ws simulations with 40 mM and $150 \mathrm{mM} \mathrm{NaCl}$ compared with experiments without additional salt (this work) and with $150 \mathrm{mM} \mathrm{NaCl}$ (from ref. 16). 

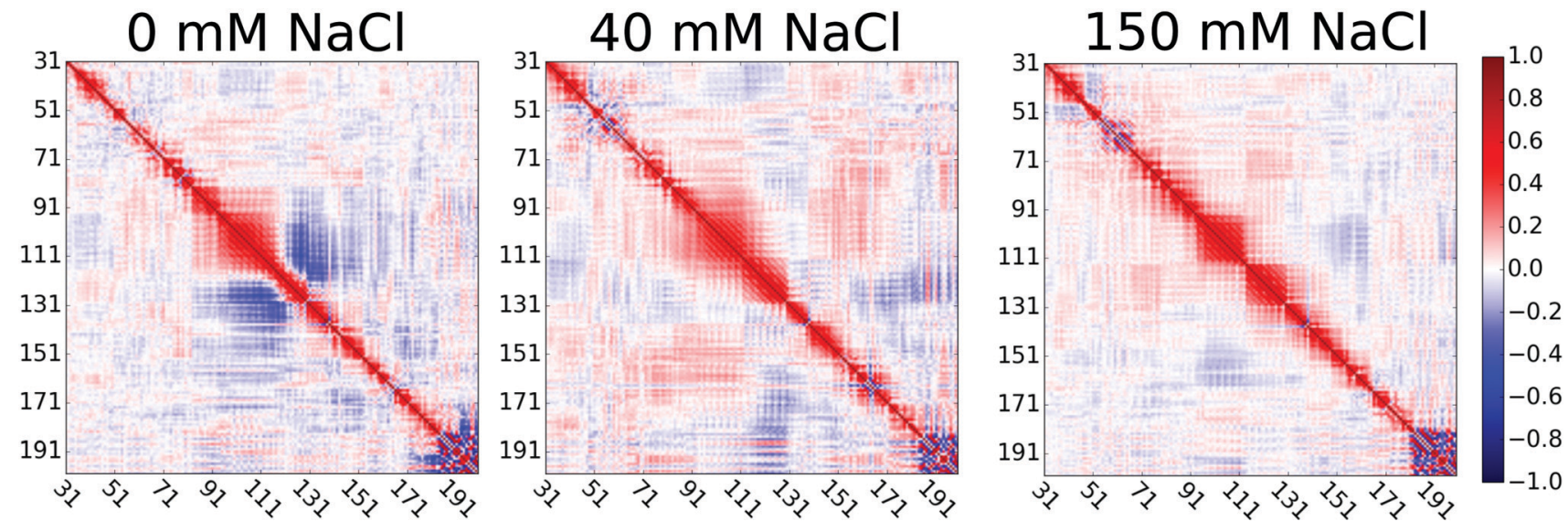

Fig. 3 Directional correlations of vectors between $C^{\alpha}$ carbons in consecutive backbone residues (31-200) in the proline rich disordered linker region of $\mathrm{HpTonB}_{30-285}$ at different concentrations of salt in solution from Amber ff03ws simulations.

screened attractive interactions between oppositely charged residues in the proline rich linker of $H p$ TonB. This is demonstrated in Fig. 3 by showing the directional correlations of vectors between $\mathrm{C}^{\alpha}$ carbons in consecutive backbone residues in the disordered linker region (residues 31-200). Negative correlations between residues $95-120$ and $120-140$ in simulation without additional salt can be explained by the hairpin-like turning of the linker due to electrostatic attraction between residues $97-113$, bearing a total charge of +9 from lysines, and residues 131-137, bearing a total charge of -2 from glutamic acids. The addition of $40 \mathrm{mM} \mathrm{NaCl}$ screens this attraction, thereby straightening the chain and eliminating the negative directional correlations. Our results suggest that the presence of ionic strength corresponding to the typical buffer concentrations used in experiments significantly screens the electrostatic interactions between charged residues in disordered proteins, thereby affecting their conformational ensembles. This should be taken into account when performing MD simulations that mimic the experimental conditions. However, further increase of ion concentration had much smaller effect on directional correlation, spin relaxation times, and conformational ensemble.

To test our approach also using other protein than $H p$ TonB, we ran simulations of partially disordered region (residues 143-259) of chicken EN2 for which the experimental spin relaxation times measured with multiple magnetic fields are available in the literature. ${ }^{28}$ In Amber ff03ws simulation of EN2 without additional salt, the oppositely charged residues between $\mathrm{N}$-terminal and $\mathrm{C}$-terminal ends interact and the protein forms a loop-like conformational ensemble (Fig. 4A). Similarly to the TonB simulation without additional salt, $T_{1}$ times measured with $800 \mathrm{MHz}$ magnet are slightly overestimated in this simulation (Fig. 4C), indicating that the loop-like structure rotates too slowly. Furthermore, residues 151-166 in the $\mathrm{N}$-terminal end form an artificial $\alpha$-helix, which leads to a poor agreement with experimental spin relaxation times in this region. Because serine and aspartatic acids have overestimated tendencies to form $\alpha$-helices in this force field, ${ }^{33}$ we mutated serine $(148,150,153,154,155,156)$ and aspartic acid (152 and 154) residues in the N-terminal end to threonine and glutamine, respectively. This mutated protein was then simulated with $40 \mathrm{mM} \mathrm{NaCl}$ to mimic the electrostatic conditions in experiments where $40 \mathrm{mM}$ sodium succinate buffer was used. ${ }^{28}$ As observed for $H p$ TonB, the attraction between charged residues is screened by the addition of salt and more extended conformational ensemble is observed (Fig. 4B). Together with the unfolding of artificial $\alpha$-helix in residues 151-166, this leads to the improved agreement with experimental spin relaxation data (Fig. 4C). Also the results from different magnetic field strengths support this conclusion (Fig. S1 in the ESI $\dagger$ ).

Our results for $H p$ TonB and EN2 demonstrate that the ${ }^{15} \mathrm{~N}$ backbone spin relaxation times are sensitive to the conformational ensembles of partially disordered proteins, and can be therefore used to evaluate MD simulations of such proteins against NMR experiments. Notably, the differences in chemical shifts (calculated using SPARTA $+^{34}$ or Shift $^{35}$ ) between simulations with different force fields that predict distinct conformational ensembles for $H p$ TonB (Fig. 1B) were not large enough to evaluate the simulations against experiments (Fig. S2, ESI $\dagger$ ). The Amber ff03ws force field captured the essential features of conformational ensembles for both partially disordered proteins studied here when the ionic strength of solvent corresponded to the experimental conditions. However, the known caveats of this force field, such as overestimated tendency of serine and aspartic acid to form $\alpha$-helices and underestimated stability of folded structures, ${ }^{19,33}$ may limit its wider usage. In conclusion, our results not only emphasize the necessity of developing better force fields for disordered protein regions, but also demonstrate that the backbone ${ }^{15} \mathrm{~N}$ spin relaxation times are good measures to evaluate conformational ensembles predicted by the force fields.

\section{Rotational motions in partially disordered proteins}

Rotational dynamics of folded proteins is typically analyzed from spin relaxation data assuming a rigid body motion for overall rotation and independent internal motions for each residue. ${ }^{36-39}$ 

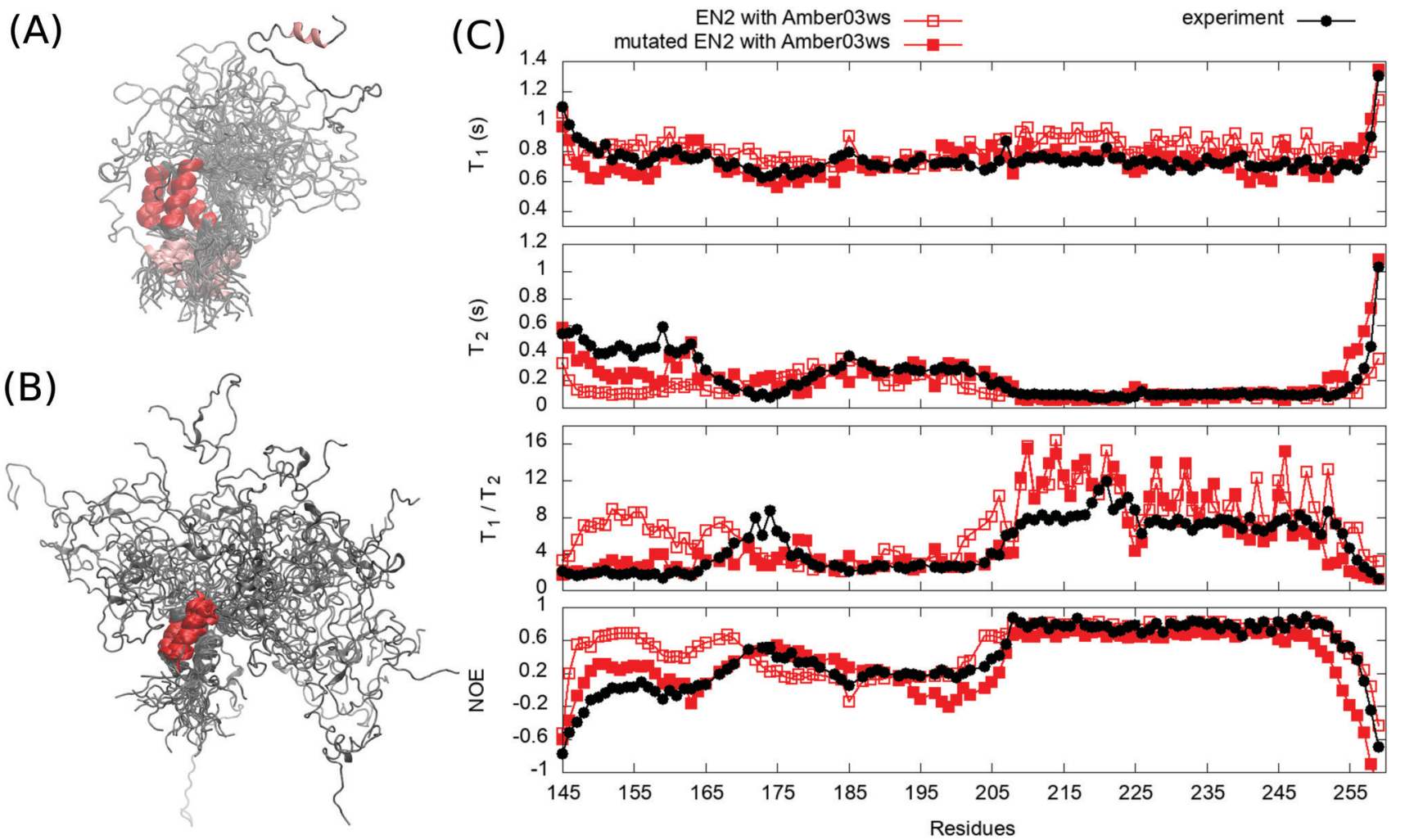

Fig. 4 Overlayed snapshots from (A) EN2 simulation without additional salt and (B) from mutated EN2 simulation with $40 \mathrm{mM} \mathrm{NaCl}$. (C) Spin relaxation times from simulations compared with experiments measured in $800 \mathrm{MHz} .{ }^{28}$ Spin relaxation times with different magnetic field strengths are shown in Fig. S1 (ESI†). Error bars of the experimental data are smaller than the point size.

Folded proteins exhibit rigid body overall rotational dynamics also in MD simulations and inaccuracies arising from incorrect viscosity of water models can be corrected during spin relaxation time calculations by scaling the overall rotational diffusion coefficients. ${ }^{15,17,40}$ Because the rigid body approximation is obviously invalid for disordered protein regions, different approaches to analyze rotational motions of disordered proteins have been proposed. ${ }^{13}$ However, the number and nature of rotational processes occurring in disordered protein regions remains unclear. $^{22,28,41,42}$

Because our $H_{p}$ TonB $_{30-285}$ simulations with Amber ff03ws force field parameters give spin relaxation times in good agreement with experiments when the ionic strength of solvents match (Fig. 2), we analyzed the underlying rotational timescales of both folded and disordered regions from these simulations using the regularized inverse Laplace transformation (ILT), ${ }^{43}$ which we used also to perform the Fourier transform of the rotational correlation functions during the spin relaxation time calculations. ${ }^{15}$ In this approach, each backbone $\mathrm{N}-\mathrm{H}$ bond rotational correlation function is written as a sum of large number of exponentials (here $N=371$ )

$$
C_{N}(t)=\sum_{i=1}^{N} \alpha_{i} \mathrm{e}^{-t / \tau_{i}},
$$

where the fixed relaxation times, $\tau_{i}$, cover the expected timescales in the system (here $10 \mathrm{ps}<\tau_{i}<50 \mathrm{~ns}$ with logarithmic spacing).
The weights of the timescales, $\alpha_{i}$, are then solved by fitting the eqn (1) to the rotational correlation functions calculated from simulations. While interpretation of $\tau_{i}$ as the relaxation times of dynamical processes present in the system is clear, the dimensionless $\alpha_{i}$ coefficients present relative weights of these processes and their physical interpretation is more complicated. ${ }^{44}$ This approach is free from a priori assumptions about the amount of underlying timescales or their relaxation times, but those are suggested by the result of the fitting procedure. However, because the solution of ILT is not unique, the resulting values must be considered as a plausible qualitative interpretation of the experimental spin relaxation data.

For folded proteins, the timescales related to the overall rigid body rotation dominate the rotation processes with the weight factors between $0.5-0.9$ in almost all residues of

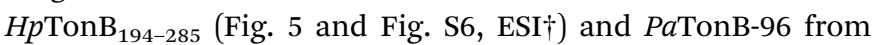
Pseudomonas aeruginosa $^{15}$ (Fig. S7, ESI $\dagger$ ). Intermediate (1-4 ns) and very fast (10 ps or faster) timescales have small contributions with the weight factors between 0.1-0.3 in the Amber ff03ws simulation of folded $H p$ TonB $_{194-285}$ (Fig. 5). In Amber ff99SB-ILDN simulations of folded $H p$ TonB $_{194-285}$ and $P a$ TonB-96 proteins, the very fast timescales have similar contribution, but intermediate timescales are observed only for few residues (Fig. S6 and S7, ESI $\dagger$ ). Therefore, our MD simulations suggest that the two-state (also known as Lipari-Szabo) model ${ }^{36,37}$ with independent overall and internal rotation timescales is a 
(A) HpTonB $194-285$

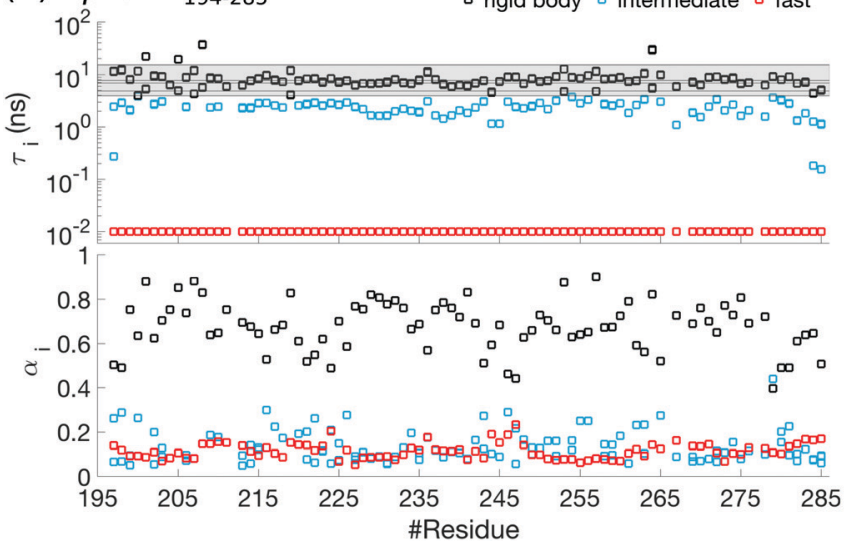

(B) $H_{p T O n B} 30-285$

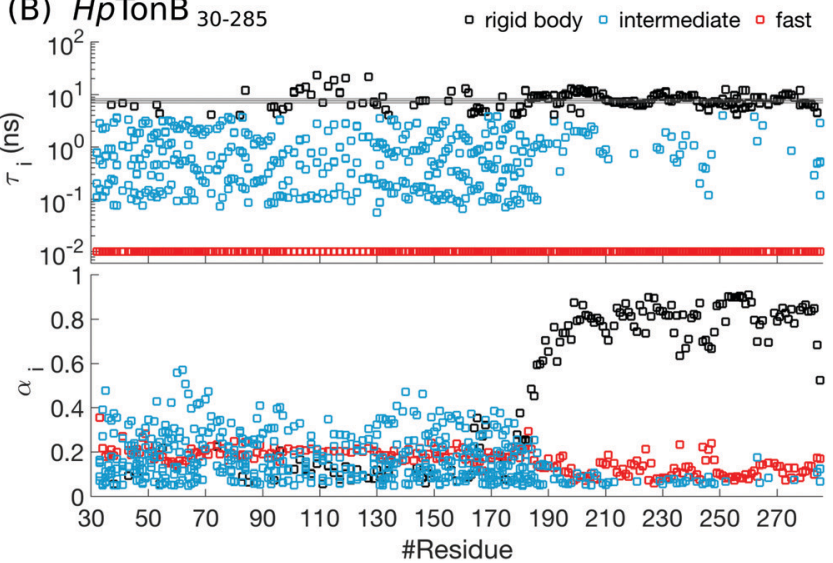

Fig. 5 Rotational timescales (top) and related weight coefficients (bottom), defined in eqn (1), from Amber ff03ws simulation of (A) a folded $\mathrm{HpT}$ TonB $194-285$ and $(\mathrm{B})$ a partially disordered $H p \mathrm{TOnB}_{36-285}$ protein. Only timescales with the weight coefficients larger than $\alpha_{i}>0.05$ are shown. Black horizontal lines and the shaded region between the extreme values display timescales from rigid body rotational diffusion coefficients. To guide the eye, the timescales above $\tau_{i} \approx 4 \mathrm{~ns}$ (the fastest rigid body timescale observed for folded HpTonB ${ }_{194-285}$ protein) are labeled as rigid body motions (black), the timescales corresponding to the saving frequency of coordinates in simulations ( $\tau_{i}=10 \mathrm{ps}$ ) are labeled as fast (red), and the timescales in between (10 ps $<\tau_{i}<4$ ns) are labeled as intermediate (turquoise).

reasonable approximation for rotational dynamics of folded proteins, even though a third timescale with small weight is observed in simulations with Amber ff03ws force field. Moreover, the $\alpha_{i}$ coefficients for rigid body motions approximately corresponds the Lipari-Szabo order parameters ${ }^{37}$ in folded regions.

Most residues in the folded region (residues 185-285) of partially disordered $H p T^{2} B_{30-285}$ exhibit similar distribution of rotational timescales as observed in globular proteins: timescales corresponding to rigid body motions dominate, while very fast and intermediate timescales have minor contributions (Fig. 5). Encouraged by this observation, we calculated the rotational diffusion coefficients of folded domains having linkers with different lengths attached. Even though the mean square angle deviations around inertia axes exhibited some non-linear features with increasing linker length (see ESI $\dagger$ ), we think that the estimated rotational diffusion coefficients and related timescales can be used to analyze the influence of a disordered linker on rotational dynamics of the folded region. As expected, the rotation of a folded region slows down upon the increasing length of attached linker (Table 2). However, the overall dynamics of folded domain in $H p \mathrm{TonB}_{179-285}$ is only slightly faster than in $H p T{ }^{2} B_{30-285}$, even though the latter protein has 2.34 times more residues. This is in line with the small changes in experimental spin relaxation times upon increasing the length of linker observed in our previous work. ${ }^{16}$ These results suggest that the linker is very flexible and that the rotational dynamics of the folded C-terminal domain is almost independent of the attached flexible disordered linker. This conclusion is supported by the similar results for overall timescales estimated from experimental $T_{1} / T_{2}$ ratios and from MD simulation analysis in Table 2 . The analysis using $T_{1} / T_{2}$ ratio assumes an isotropic rigid body rotational diffusion for protein,${ }^{45}$ while MD simulation analysis takes anisotropic effects fully into account. ${ }^{15}$ Similarity of the results from these two approaches suggests that the assumption of rigid body rotational diffusion is relatively reasonable for folded domains even with long disordered linkers attached.

Disordered region in $H p \mathrm{TonB}_{30-285}$ (residues 30-184) exhibits clearly different distribution of rotational motions than the folded region, having dispersed timescales between approximately $60 \mathrm{ps}$ and $30 \mathrm{~ns}$ without any dominating timescales (Fig. 5). Similar differences in distribution of timescales between folded and disordered regions are seen also in $H p T^{T o n B} B_{30-285}$ with $150 \mathrm{mM}$ $\mathrm{NaCl}$ (Fig. S8, ESI $\dagger$ ) and EN2 (Fig. S9, ESI $\dagger$ ), although intermediate timescales are more pronounced in the folded region of EN2, most likely due to the less rigid structure than in $H p$ TonB simulations. The large number of detected timescales in disordered regions in both proteins suggests that the models assuming two or three timescales for rotational motions are too simple to capture all relevant rotational processes in disordered proteins. This is in line with the recent study where dihedral rotation and rotational tumbling motions of peptide

Table 2 Rotational diffusion coefficients $\left(\mathrm{rad}^{2} \cdot 10^{7} \mathrm{~s}^{-1}\right.$ ) of the folded C-terminal domain (residues 196-280) of HpTonB with different lengths of disordered linker attached from spin relaxation experiments interpreted using MD simulations. The estimates of overall rotational timescales from experimental $T_{1} / T_{2}$ ratio, $\tau_{\mathrm{c}}^{T_{1} / T_{2}}$, are taken from our previous work ${ }^{16}$

\begin{tabular}{lcllll}
\hline & $D_{x}$ & $D_{y}$ & $D_{z}$ & $D_{\mathrm{av}}$ & $\tau_{\mathrm{c}}(\mathrm{ns})$ \\
\hline HpTonB $_{194-285}$ & $1.9 \pm 0.3$ & $2.3 \pm 0.2$ & $4.1 \pm 0.1$ & $2.8 \pm 0.2$ & $5.9 \pm 0.5$ \\
HponB $_{179-285}$ & $1.86 \pm 0.05$ & $2.4 \pm 0.2$ & $3.5 \pm 0.3$ & $2.6 \pm 0.2$ & $6.4 \pm 0.5$ \\
HpTonB $_{36-285}$ & $1.80 \pm 0.01$ & $2.1 \pm 0.2$ & $2.4 \pm 0.3$ & $2.1 \pm 0.2$ & $7.9 \pm 0.8$
\end{tabular}


planes were found to have multiple timescales. ${ }^{42}$ Because large number of parameters are needed to describe such dynamics, the multiple timescale dynamics is very difficult to detect even with the excessive amount of experimental data measured at different conditions. $^{28,41}$ Khan et al. have analyzed rotational timescales of EN2 by fitting a sum of exponential correlation functions with six fixed timescales to the experimental data detected at different magnetic fields. ${ }^{28}$ In line with our results (Fig. S9, ESI $\dagger$ ), timescales between $\sim 10$ ps and $\sim 1$ ns were not present in the folded region in their analysis. However, their dominant timescale, $5.27 \mathrm{~ns}$, is shorter than $\sim 10 \mathrm{~ns}$ in our results, and they observed significant weight for a very long $21 \mathrm{~ns}$ timescale, which is not present in our results. For disordered region of EN2, Khan et al. observed dispersed timescales between 21 ps and 21 ns which is in line with our results. However, the timescale of $1.33 \mathrm{~ns}$ dominated the rotation of disordered region in their analysis, while we did not detect any dominant timescale in the disordered region. We believe that these differences may arise because the dynamical model with six fixed timescales that are equal for all residues may be too simple for partially disordered proteins. Our MD simulations suggest that disordered protein regions have more complex distributions of rotational timescales with multiple components that vary between the residues. While this distribution of timescales is very difficult to detect by fitting to even a vast amount of experimental data, MD simulations with realistic force field parameters can capture the relevant rotational processes detected by spin relaxation times for disordered protein regions, thereby giving an interpretation of experimental data with unprecedented details.

\section{Implications to TonB function}

TonB proteins are part of TonB complexes, which propagate energy generated by the proton-motive force in the inner membrane of Gram-negative bacteria to the TonB dependent receptors (TBDTs) in the outer membrane with unknown mechanism. ${ }^{25}$ Because TBDTs use this energy to transport vital nutrients inside bacteria, TonB is a potential target for antibiotics. N-terminal domain of TonB protein is anchored in the inner membrane and the proline rich linker region is expected to span across the approximately $20 \mathrm{~nm}$ wide periplasmic space to enable the C-terminal domain to interact with TBDTs in the outer membrane ${ }^{25,46-48}$ (Fig. 6). The folded domains of proteins in TonB complex have been characterized using cryo-EM, X-ray crystallography, NMR, single molecule experiments and MD simulations. ${ }^{16,29,49-57}$ However, the role of disordered proline rich linker in TonB function (residues 36-184 in HpTonB, Fig. 1) is poorly understood due to the lack of robust methods to characterize such protein domains. Electron paramagnetic resonance (EPR) and early NMR experiments suggest that the TonB linker in $E$. coli is disordered but still relatively rigid due to abundant polyproline helices, ${ }^{47,58,59}$ while some studies assume a completely rigid structure that would enable the mechanical energy transfer across periplasm via rigid rotation. ${ }^{50}$

In the conformational ensemble of $H p$ TonB $_{36-285}$ in solution with physiological ion concentration (Fig. 2), resolved here using Amber ff03ws simulations and NMR spin relaxation

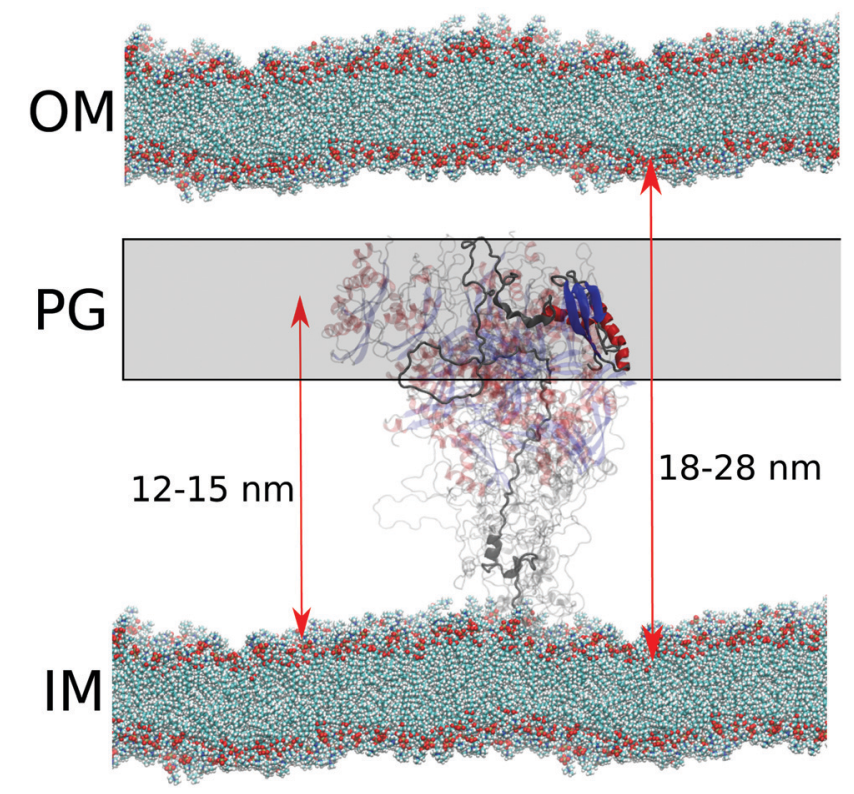

Fig. 6 Schematic figure of conformational ensemble sampled by periplasmic TonB in sub-microsecond timescales in cell envelope of Gram-negative bacteria. Distances from the inner membrane (IM) to the outer membrane (OM) and proteoglycan (PG) layer are from ref. 46.

experiments, the proline rich linker is very flexible with the rotational timescales faster than $30 \mathrm{~ns}$ (Fig. 5) and the folded C-terminal domain rotates almost independently of the linker region (Table 2). On the other hand, significant fractions of polyproline helices (PPII) with the probability up to approximately $70 \%$ were observed in the linker region in our simulations (Fig. S17, ESI $\dagger$ ). This is in line with the previous NMR and CD measurements of TonB sequence from $E$. coli. ${ }^{47,58,59}$ Further data supporting the relatively rigid polyproline helix conformations of the TonB linker in E. coli comes from DEER EPR experiments, where distances between spin labels at different locations in the linker were measured. ${ }^{47}$ The EPR experiments give average length of $4.6 \pm 0.1 \mathrm{~nm}$ for the residues 88-106 in E. coli TonB consisting mainly of PK repeats. The corresponding sequence in HpTonB between residues 94-112 (Fig. S16B, ESI $\dagger$ ) has the same average length of $4.6 \pm$ $0.2 \mathrm{~nm}$ in the Amber ffo3ws simulation with $40 \mathrm{mM} \mathrm{NaCl}$. In conclusion, the conformational ensemble from Amber ff03ws simulations (Fig. 2) is in line with the experimental spin relaxation data, as well as with the interpretation of previous NMR and EPR experiments from $E$. coli sequence, ${ }^{47,58,59}$ suggesting that the linker is disordered but has relatively extended conformation with high propensity of polyproline helices.

Because crystal structures suggest that TonB interacts with TBDTs via the $\beta 3$ strand in the folded C-terminal domain of TonB (residues $272-281$ in $H p$ TonB), ${ }^{5,54}$ the linker domain should reach across the approximately $20 \mathrm{~nm}$ wide periplasmic space $^{46}$ to bring the C-terminal domain into contact with TBDTs in outer membrane (Fig. 6). In our conformational ensemble, resolved from Amber ff03ws simulations in $40 \mathrm{mM}$ $\mathrm{NaCl}$ solution (Fig. 2), the maximum distance between the 
$\beta 3$ strand atoms and the end of transmembrane domain (residue 30) is $15.2 \mathrm{~nm}$ with the average value of $11.9 \pm$ $0.3 \mathrm{~nm}$, which is close to the typical distance between inner membrane and proteoglycan layer in Gram-negative bacteria, but shorter than the width of periplasmic space (Fig. 6).

In summary, our results suggest that the periplasmic part of TonB exhibits rapid conformational fluctuations with the timescales faster than $30 \mathrm{~ns}$ and that the C-terminal domain locates on average close to the proteoglycan layer (Fig. 6). These results are in line with the previously proposed models where the C-terminal domain of TonB reaches only to the proteoglycan layer, while the binding site in TBDTs extends into periplasm to interact with the $\beta 3$ helix in CTD of TonB at the proteoglycan layer. ${ }^{50,60}$ Alternatively, the proton-motive force could be used to increase the linker length to push the C-terminal domain of TonB to interact with the TBDTs in the outer membrane, and the relaxation back to the equilibrium length would then mechanically open the TBDT. On the other hand, the high flexibility of the linker and uncorrelated rotational motions of linker and the folded C-terminal domain do not support models where rotational torque is mediated across periplasmic space via rigid linker. ${ }^{50}$ Also scanning of TBDTs directly from the outer membrane ${ }^{48}$ seems less likely due to the short average length of the linker. However, we cannot exclude the possibility that changing $\mathrm{pH}$ conditions, TonB dimerization or its interactions with membrane, proteoglycan layer or other proteins in TonB complex would increase the length or rigidity of the linker. We believe that MD simulations with realistic conformational ensembles of disordered linkers, evaluated using experimental spin relaxation times, paves the way for further studies to understand the function of TonB complex, as well as other biological machineries where disordered protein regions play crucial roles.

\section{Conclusions}

Our results show that the backbone ${ }^{15} \mathrm{~N}$ spin relaxation times are sensitive not only to the protein dynamics at multiple timescales, but also to the conformational ensemble of partially disordered proteins. Using this feature, we evaluated the conformational ensembles of partially disordered $\mathrm{HpTonB}$ protein from MD simulations with different force fields against experimental spin relaxation time data. Simulations with Amber ffo3ws force field ${ }^{32}$ predicted the conformational ensemble of periplasmic domain in $H p$ TonB when the ionic strength of solvent corresponded the experimental conditions. The presence of sufficient amount of ions was necessary because they screen electrostatic interactions between charged residues, thereby having a substantial influence on conformational ensemble. These conclusions were further supported by the simulations of EN2 protein compared with previously published spin relaxation data. ${ }^{28}$

MD simulations that reproduced the experimental spin relaxation times revealed that the timescales from overall rigid body rotation dominate in fully folded proteins and folded regions in partially disordered proteins, while very fast (10 ps or faster relaxation times) and intermediate (1-4 ns relaxation times) timescales give small contributions. Furthermore, the rotational dynamics of folded region was almost independent of the attached disordered linker. Therefore, the estimation of overall rotational timescales from experimental $T_{1} / T_{2}$ ratio using simple model assuming isotropic rotation ${ }^{45}$ gave reasonable results even for folded region with long disordered linker attached. In contrast, disordered regions exhibit rotational dynamics with multiple dispersed timescales between 60 ps and 30 ns without any dominating contribution, suggesting that the dynamical modes of disordered proteins are very difficult to capture by fitting to even a large amount of experimental data. We suggest that the dynamical modes of disordered protein regions can be captured using MD simulations with accurate force field and solvent ionic strength matching the experimental conditions.

The resolved conformational ensemble of $\mathrm{HpTonB}_{36-285}$ is in line with the previous NMR and EPR studies ${ }^{47,58,59}$ of TonB linker from $E$. coli, but gives unprecedented details of periplasmic domain of TonB that helps to understand its function. In our results, the equilibrium length of the TonB linker is not sufficient to reach across the periplasmic space, suggesting that either TBDTs has to extend the periplasmic space, ${ }^{50,60}$ or the length of linker has to increase, for example, due to changing $\mathrm{pH}$ conditions or interactions with other molecules. One possible scenario is that the proton-motive force would be used to induce such increase in the linker length. The energy released by the relaxation of linker to its equilibrium length could be then used to transport nutrients through the TBDT. Our results pave the way for MD simulations that could be combined with experimental approaches to test this, and other hypotheses on the functional mechanism of TonB complex function.

Using MD simulations to interpret the conformational ensembles and dynamics of disordered protein regions from NMR data has two main advantages over the other available methods. ${ }^{11-13,41,61}$ First, MD simulations that correctly predict the spin relaxation times can be used to interpret the experiments without a priori assumptions about the amount of rotational timescales. Second, MD simulation force fields that give correct conformational ensembles for disordered protein regions can be straightforwardly used to model these regions within complex biomolecules assemblies under various conditions. For example, our results suggest that TonB complex could be simulated by combining the Amber ff03ws force field with Amber compatible lipid force fields $s^{62,63}$ in order to fully understand its function. However, applications of Amber ff03ws force field are limited by its problems to predict the stability of $\alpha$-helices in some situations. ${ }^{19,33}$ Therefore, our results also emphasize the necessity of force field development to correctly capture the conformational ensembles of partially disordered proteins in various conditions. Our results suggest that the backbone ${ }^{15} \mathrm{~N}$ spin relaxation times are suitable experimental references for these efforts because they are more sensitive to the conformational ensemble than, for example, chemical shifts which are often used in force field optimization or to resolve conformational ensembles of disordered proteins. ${ }^{19,64}$ 


\section{Materials and methods}

\section{MD simulations}

Hp TonB $_{30-285}$. Initial structure of the proline rich disordered linker (residues 30-178) was generated using ProBuilder (http:// 159.149.85.2/probuilder.htm) with parameters that generated almost a straight protein chain without any secondary structure. This chain was attached to the N-terminal end of the lowest energy NMR structure of $H p T^{T} B_{179-285}$ (PDB code: $6 \mathrm{SLY}$ ). ${ }^{16}$ The energy was first minimized in vacuum using Amber ff99SB$\mathrm{ILDN}^{30}$ force field parameters. Simulations with three different force fields, Amber ff99SB-ILDN, ${ }^{30}$ CHARMM $36 \mathrm{~m}^{31}$ and Amber ff03 $\mathrm{ws}^{32}$ were initiated from this structure. The protein was solvated with 360464 TIP3P ${ }^{65}$ water molecules for the Amber ff99SB-ILDN $^{30}$ simulation, with 365523 charmm version of TIP3P water molecules for the CHARMM36m simulation, and with 364657 TIP4P/2005 water molecules ${ }^{66}$ for the Amber ff03ws simulation. In addition, all the systems contained six chloride ions to retain the electroneutrality. Parallelepiped shaped box was used in all simulations to minimize the required amount of water.

The protein significantly collapsed in both Amber ff99SBILDN and CHARMM36m simulations during $40 \mathrm{~ns}$ and $120 \mathrm{ns,}$ respectively. Due to the reduced size of the protein, the amount of water molecules were reduced to 45950 and 35892 in these simulations, respectively. The simulation were then continued to have total lengths of $384 \mathrm{~ns}$ and $520 \mathrm{~ns}$, respectively. The last $280 \mathrm{~ns}$ and $400 \mathrm{~ns}$ were used in the analysis, respectively. The size of protein in the Amber ffo3ws simulation was much less reduced and the amount of water molecules was reduced to 123497 after $5.3 \mathrm{~ns}$ simulation. This system was then simulated $1171 \mathrm{~ns}$ and the last $1000 \mathrm{~ns}$ was used in the analyzes. The temperature was $310 \mathrm{~K}$ in Amber ff99SB-ILDN and CHARMM36m simulations and $298 \mathrm{~K}$ in the Amber ffo3ws simulation. Simulations were ran using Gromacs versions between 5.0 and 2020 series. ${ }^{67}$ Rest of the simulation details are described in the ESI. $\dagger$ Simulation files and trajectories are available from ref. 68-70.

Sodium and chloride ions described with parameters containing the electronic continuum correction (ECC), ${ }^{71,72}$ available from https://bitbucket.org/hseara/ions/, were added into the Amber ffo3ws simulation to study the influence of solvent ionic strength on conformational ensemble. The 6 chloride counterions were described by the default Amber ffo3ws parameters. Because the protein immediately expanded upon addition of $150 \mathrm{mM} \mathrm{NaCl}$ and started to interact with its own periodic image after 200 ns simulation, the number of water molecules was increased for simulations containing ions. Simulation containing $150 \mathrm{mM} \mathrm{NaCl}$ had 261780 water molecules with $711 \mathrm{Na}^{+}$and $\mathrm{Cl}^{-}$ ions, and simulation containing $40 \mathrm{mM} \mathrm{NaCl}$ had 262822 water molecules with $190 \mathrm{Na}^{+}$and $\mathrm{Cl}^{-}$ions. Simulation files and trajectories with $\mathrm{NaCl}$ are available from ref. 73 and 74.

$\boldsymbol{H p}$ TonB $_{179-285}$. $H p$ TonB $_{179-285}$ was simulated $600 \mathrm{~ns}$ using Amber ff99SB-ILDN ${ }^{30}$ force field with 8894 TIP4P $^{65}$ water molecules and 390 ns using Amber ff03ws force field ${ }^{32}$ with 9586 TIP4P/2005 water molecules at $303 \mathrm{~K}$. Two potassium or sodium ions, respectively, were added to keep the system electroneutral.
Lowest energy NMR structure of $H p$ TonB $_{179-285}$ (PDB code: $6 \mathrm{SLY})^{16}$ was used as the initial structure. Equilibration of the trajectories were followed by monitoring the protein RMSD, inertia tensor eigenvalues and rotation angles. The first $150 \mathrm{~ns}$ and $10 \mathrm{~ns}$, respectively, were omitted from the trajectory to remove the significant fluctuations in these parameters. Simulations were ran using Gromacs versions between 5.0 and 2020 series. ${ }^{67}$ The trajectories and the related simulation files are available from ref. 75 and 76.

Hp TonB $_{\text {194-285. }}$. Hp TonB ${ }_{194-285}$ was simulated 1120 ns using Amber ff03ws force field ${ }^{32}$ with 9101 TIP4P/2005 water molecules at $298 \mathrm{~K}$. Lowest energy NMR structure of $H p$ TonB $_{194-285}$ (PDB code: $5 \mathrm{LW} 8)^{29}$ was used as the initial structure. First $10 \mathrm{~ns}$ was omitted from the trajectory in the analysis. Simulation was ran using Gromacs 2019. ${ }^{67}$ The trajectories and the related simulation files are available from ref. 77 .

Chicken EN2. The sequence of EN2 was obtained from BMRDB (http://www.bmrb.wisc.edu/) with the accession number 17325. The initial structure of the disordered part (residues 143-194) was generated using ProBuilder (http://159. 149.85.2/probuilder.htm) with the parameters that produced almost a straight amino acid chain with no secondary structure. The rest of the molecule (residues 195-259) including the structured homeodomain region was prepared using the NMR structure of chicken Engrailed 2 (PDB code: $3 \mathrm{ZOB})^{78}$ by mutating the first five residues remaining from the cleavage into asparagine, proline, asparagine, lysine and glutamic acid with a MODELLER script (https://salilab.org/modeller/wiki/ Mutate\%20model). The last two residues of the chain were removed. The disordered chain was then attached to the $\mathrm{N}$-terminal end of the folded part. The protein was solvated with 57289 TIP4P/2005 water molecules and 12 chloride ions and simulated $1300 \mathrm{~ns}$ at $310 \mathrm{~K}$ using Gromacs 2019 and Amber ff03ws force field. The first $300 \mathrm{~ns}$ were discarded from the analysis. The trajectories and the related simulation files are available from ref. 79 .

Mutated chicken EN2 with $40 \mathrm{mM} \mathrm{NaCl}$. Because serine and aspartic acid residues overestimate the tendency to form $\alpha$-helices in Amber ffo3ws force field, ${ }^{33}$ we run a simulation of chicken EN2 where serine $(148,150,153,154,155,156)$ and aspartic acid $(152,154)$ residues in the $\mathrm{N}$ terminal end were mutated to threonine and glutamine, respectively. This was done using a MODELLER script (https://salilab.org/modeller/ wiki/Mutate\%20model). The initial structure was taken from the original EN2 simulation after $300 \mathrm{~ns}$. This was solvated with 57215 TIP4P/2005 water molecules, 12 chloride counterions and 41 sodium and chloride ions to make the solution concentration correspond $40 \mathrm{mM} \mathrm{NaCl}$. The simulation was run for 1080 ns using Gromacs 2019 and Amber ff03ws force field. The last $1000 \mathrm{~ns}$ was used in the analysis. The trajectories and the related simulation files are available from ref. 80 .

Calculation of spin relaxation times. The ${ }^{15} \mathrm{~N}$ backbone spin relaxation times were calculated as described previously. ${ }^{15}$ The rotational correlation functions were calculated using Gromacs command gmx rotacf and the programs used to calculate the spin relaxation times are available from ref. 81. 
In our previous study, we showed that the underestimated viscosity of TIP3P water model can be corrected during spin relaxation time calculation by dividing the rotational diffusion coefficients of folded proteins with a constant factor of 2.9. ${ }^{15}$ Here, we apply the same approach to the folded C-terminal domain of $H p$ TonB $_{30-285}$ when calculating the spin relaxation times from Amber ff99SB-ILDN and CHARMM36m simulations, where TIP3P water was used. For this, we first calculate the rotational diffusion coefficients of the folded C-terminal end (residues 196-280). The rotational correlation functions from $\mathrm{N}-\mathrm{H}$ bonds of residues 175-285 were then divided to the internal and overall motions, and the contribution of overall motion was corrected during spin relaxation time calculation by dividing the diffusion coefficients with a factor 2.9 as described in our previous work. ${ }^{15}$ The water viscosity correction was not applied to the disordered linker region because it does not rotate as a rigid body and is therefore expected to be less affected by the fluid viscosity. ${ }^{82}$ Because spin relaxation times from Amber ff03ws force field are in good agreement with experiments for the folded $H p$ TonB $_{194-285}$ protein without any correction for the water viscosity (Fig. S3 in the ESI $\dagger$ ), the spin relaxation times from Amber ff03ws simulations of $\mathrm{HpTonB}_{36-285}$ and EN2 protein were calculated directly from simulation data without any corrections for water viscosity.

Correlation functions up to one hundredth of the simulation time should give a sufficient statistics for the analysis of rotation from a single molecule MD simulation. ${ }^{83}$ We observed that insufficient statistics in correlation functions induce artificial timescales with the longest possible timescale (50 ns) when fitting the correlation function to eqn (1). Therefore, when calculating spin relaxation times directly from Amber ff03ws simulations without using diffusion coefficients, we selected the time frames to be analyzed from correlation functions such that the number of weights above 0.05 for artificial $50 \mathrm{~ns}$ timescale is minimized. The analyzed time frames from correlation functions were the first $15 \mathrm{~ns}$ for $H p$ TonB $_{194-285}$, the first $10 \mathrm{~ns}$ for $\mathrm{HpTonB}_{30-285}$ without $\mathrm{NaCl}$, and the first $25 \mathrm{~ns}$ for $H p$ TonB $_{30-285}$ with $\mathrm{NaCl}$ and EN2.

Quantitative error analysis of calculated spin relaxation times is complicated because each step in the analysis has different sources of errors. However, independent simulation replicas of folded $P a$ TonB-96 protein in our previous work ${ }^{15}$ gives an average deviations of $\Delta T_{1}=0.06 \mathrm{~s}, \Delta T_{2}=0.02 \mathrm{~s}$, and $\Delta \mathrm{NOE}=0.04$, which can be considered as the estimates of accuracy of the calculated values.

Calculation of rotational diffusion coefficients. Rotational diffusion coefficients around protein inertia axes were calculated as in our previous work. ${ }^{15}$ For folded $H p$ TonB $_{194-285}$ and $H p$ TonB $_{179-285}$, the average values from simulations with two different force fields are reported (ref. 15 and Fig. S3-S5, ESI $\dagger$ ), and the error bars are the differences between force fields divided by two. For folded region in partially disordered ${ }_{H p}$ TonB $_{30-285}$ protein, mean square angle deviation exhibits some non-linear behavior (Fig. S13 and S14, ESI $\dagger$ ). Therefore, the slopes of mean square angle deviations between 0-10 ns and 5-10 ns were calculated, and the average values of these were reported. The error bars are the differences between these values divided by two. The rotational timescales $\left(\tau_{i}\right.$ in eqn (1)) related to the rigid body rotational diffusion were calculated using eqn (9) in ref. 15.

Analysis of backbone orientational correlation. To analyze the orientational correlation between different residues in protein backbone, we calculated the dot products of vectors connecting the $\mathrm{C}^{\alpha}$ carbons in neighboring residues, $\left\langle v_{i} \cdot v_{j}\right\rangle$, where $v_{i}$ is normalized vector between $\mathrm{C}_{i}^{\alpha}$ and $\mathrm{C}_{i+1}^{\alpha}$, and the ensemble average is taken over the trajectory. The positive or negative values of the averaged and normalized dot product indicate positive or negative orientational correlation between the residues, respectively. Completely random orientations give zero.

\section{NMR spin relaxation time experiments}

Using segmental labeling with salt-inducible split intein as described in our previous work, ${ }^{16}$ we prepared $H p$ TonB $_{36-285}$ with residues $36-154{ }^{15} \mathrm{~N}$ labeled, as well as $H p$ TonB $_{36-285}$ bearing K154R mutation with residues $155-285{ }^{15} \mathrm{~N}$ labeled. This segmental labeling reduces the overlap of NMR peaks and enables the measurement of spin relaxation times of almost all residues in large partially disordered proteins. ${ }^{16}$ Spin relaxation times were measured using $5 \mathrm{~mm}$ Shigemi tubes filled with $1.37 \mathrm{mM}$ concentration of $H_{p}$ TonB $_{36-285}$ with residues 36-154 labeled, or $1.56 \mathrm{mM}$ concentration of $H p$ TonB $_{36-285}$ with residues 155-285 labeled. Both samples were solvated with $20 \mathrm{mM}$ phosphate buffer at $\mathrm{pH} 6$ and $5 \%$ of $\mathrm{D}_{2} \mathrm{O}$. Experiments were performed on a Bruker Avance $850 \mathrm{MHz}$ spectrometer equipped with a cryogenically cooled probe head using the same pulse sequence ${ }^{45,84,85}$ and settings as previously ${ }^{16}$ with the delay times of 50,100, 200, 300, 500, 700, and $900 \mathrm{~ms}$ for $T_{1}$, and $34,68,102,136,170,204,238$, and $272 \mathrm{~ms}$ for $T_{2}$. The sample temperature in experiments was $298 \mathrm{~K}$. The spin relaxation times of $H p$ TonB $_{36-285}$ with $150 \mathrm{mM} \mathrm{NaCl}$ were measured previously. ${ }^{16}$ The $T_{1}$ and $T_{2}$ relaxation data were processed and analyzed using Bruker Dynamic Center software (version 2.5.5) and the NOE relaxation times were processed and analyzed using CcpNmr Analysis software (version 2.4.2). ${ }^{86}$

\section{SAXS experiments}

For SAXS experiments, $H p$ TonB $_{36-285}$ was prepared without isotopic labeling as described previously ${ }^{16}$ and solvated to $200 \mu \mathrm{l}$ of $20 \mathrm{mM}$ phosphate buffer at pH 6 with $150 \mathrm{mM} \mathrm{NaCl}$. Concentration of $\mathrm{HpTonB}_{36-285}$ in the sample was $0.36 \mathrm{mM}$. SAXS experiments coupled with Size-Exclusion Chromatography (SEC-SAXS) were performed at beamline B21, Diamond Light Source, UK. Radius of gyration was calculated from frames with integral of ratio to background larger than 0.255 using Guinier analysis. After discarding the largest and smallest values, the average of these values was calculated and the error bars were determined to cover all the values.

\section{Data availability}

The simulation trajectories are available from ref. 68-70, 73-77, 79, 80 and collection of derived data and computer codes from ref. 87. 


\section{Conflicts of interest}

There are no conflicts to declare.

\section{Acknowledgements}

We thank Hannes Beyer and Miska Niemelä for the sample preparation for the SAXS experiments, and Sofia Backlund for the help in processing experimental spin relaxation time data. CSC-IT center for Science Ltd, Finland, is acknowledged for the allocation of computational resources. This work was financially supported by the Academy of Finland (315596, 319902, 277335) and Sigrid Juselius Foundation. The NMR facility has been supported by Biocenter Finland and HiLIFE-INFRA. We thank Diamond Light Source (Didcot, UK) for allowing access to the BioSAXS beamline B21.

\section{Notes and references}

1 J. Ward, J. Sodhi, L. McGuffin, B. Buxton and D. Jones, J. Mol. Biol., 2004, 337, 635-645.

2 V. N. Uversky, C. J. Oldfield and A. K. Dunker, Annu. Rev. Biophys., 2008, 37, 215-246.

3 B. Xue, A. K. Dunker and V. N. Uversky, J. Biomol. Struct. Dyn., 2012, 30, 137-149.

4 Y. E. Shapiro, Prog. Biophys. Mol. Biol., 2013, 112, 58-117.

5 X. Chen, J. L. Zaro and W.-C. Shen, Adv. Drug Delivery Rev., 2013, 65, 1357-1369.

6 E. Papaleo, G. Saladino, M. Lambrughi, K. Lindorff-Larsen, F. L. Gervasio and R. Nussinov, Chem. Rev., 2016, 116, 6391-6423.

7 R. B. Berlow, H. J. Dyson and P. E. Wright, J. Mol. Biol., 2018, 430, 2309-2320.

8 N. D. Keul, K. Oruganty, E. T. Schaper Bergman, N. R. Beattie, W. E. McDonald, R. Kadirvelraj, M. L. Gross, R. S. Phillips, S. C. Harvey and Z. A. Wood, Nature, 2018, 563, 584-588.

9 D. Eliezer, Curr. Opin. Struct. Biol., 2009, 19, 23-30.

10 M. Brucale, B. Schuler and B. Samorí, Chem. Rev., 2014, 114, 3281-3317.

11 M. R. Jensen, P. R. Markwick, S. Meier, C. Griesinger, M. Zweckstetter, S. Grzesiek, P. Bernadó and M. Blackledge, Structure, 2009, 17, 1169-1185.

12 M. R. Jensen, M. Zweckstetter, J.-R. Huang and M. Blackledge, Chem. Rev., 2014, 114, 6632-6660.

13 N. Salvi, A. Abyzov and M. Blackledge, Prog. Nucl. Magn. Reson. Spectrosc., 2017, 102-103, 43-60.

14 S. Milles, N. Salvi, M. Blackledge and M. R. Jensen, Prog. Nucl. Magn. Reson. Spectrosc., 2018, 109, 79-100.

15 O. H. S. Ollila, H. A. Heikkinen and H. Iwai, J. Phys. Chem. B, 2018, 122, 6559-6569.

16 A. Ciragan, S. M. Backlund, K. M. Mikula, H. M. Beyer, O. H. S. Ollila and H. Iwaï, Front. Chem., 2020, 8, 136.

17 V. Wong and D. A. Case, J. Phys. Chem. B, 2008, 112, 6013-6024.
18 J. Huang and A. D. MacKerell, Curr. Opin. Struct. Biol., 2018, 48, 40-48.

19 P. Robustelli, S. Piana and D. E. Shaw, Proc. Natl. Acad. Sci. U. S. A., 2018, 115, E4758-E4766.

20 J. Henriques, L. Arleth, K. Lindorff-Larsen and M. Skepö, J. Mol. Biol., 2018, 430, 2521-2539.

21 K. Kämpf, S. A. Izmailov, S. O. Rabdano, A. T. Groves, I. S. Podkorytov and N. R. Skrynnikov, Biophys. J., 2018, 115, 2348-2367.

22 N. Rezaei-Ghaleh, G. Parigi, A. Soranno, A. Holla, S. Becker, B. Schuler, C. Luchinat and M. Zweckstetter, Angew. Chem., 2018, 57, 15262-15266.

23 N. Salvi, A. Abyzov and M. Blackledge, J. Phys. Chem. Lett., 2016, 7, 2483-2489.

24 Y. Xue and N. R. Skrynnikov, J. Am. Chem. Soc., 2011, 133, 14614-14628.

25 H. Celia, N. Noinaj and S. K. Buchanan, Int. J. Mol. Sci., 2020, 21, 375.

26 L. T. Peltenburg and C. Murre, EMBO J., 1996, 15, 3385-3393.

27 I. Foucher, M. L. Montesinos, M. Volovitch, A. Prochiantz and A. Trembleau, Development, 2003, 130, 1867-1876.

28 S. Khan, C. Charlier, R. Augustyniak, N. Salvi, V. Déjean, G. Bodenhausen, O. Lequin, P. Pelupessy and F. Ferrage, Biophys. J., 2015, 109, 988-999.

29 A. Ciragan, A. S. Aranko, I. Tascon and H. Iwaï, J. Mol. Biol., 2016, 428, 4573-4588.

30 K. Lindorff-Larsen, S. Piana, K. Palmo, P. Maragakis, J. L. Klepeis, R. O. Dror and D. E. Shaw, Proteins: Struct., Funct., Bioinf., 2010, 78, 1950-1958.

31 J. Huang, S. Rauscher, G. Nawrocki, T. Ran, M. Feig, B. L. de Groot, H. Grubmüller and A. D. MacKerell Jr, Nat. Methods, 2017, 14, 71-73.

32 R. B. Best, W. Zheng and J. Mittal, J. Chem. Theory Comput., 2014, 10, 5113-5124.

33 R. B. Best, D. de Sancho and J. Mittal, Biophys. J., 2012, 102, 1462-1467.

34 Y. Shen and A. Bax, J. Biomol. NMR, 2010, 48, 13-22.

35 S. Neal, A. Nip, H. Zhang and D. S. Wishart, J. Biomol. NMR, 2003, 26, 215-240.

36 H. Wennerström, B. Lindman, O. Söderman, T. Drakenberg and J. B. Rosenholm, J. Am. Chem. Soc., 1979, 101, 6860-6864.

37 G. Lipari and A. Szabo, J. Am. Chem. Soc., 1982, 104, 4546-4559.

38 D. Korzhnev, M. Billeter, A. Arseniev and V. Orekhov, Prog. Nucl. Magn. Reson. Spectrosc., 2001, 38, 197-266.

39 A. G. Palmer, Chem. Rev., 2004, 104, 3623-3640.

40 J. J. Prompers and R. Brüschweiler, J. Am. Chem. Soc., 2002, 124, 4522-4534.

41 A. Abyzov, N. Salvi, R. Schneider, D. Maurin, R. W. Ruigrok, M. R. Jensen and M. Blackledge, J. Am. Chem. Soc., 2016, 138, 6240-6251.

42 N. Salvi, A. Abyzov and M. Blackledge, Angew. Chem., 2017, 56, 14020-14024.

43 A. Nowacka, N. Bongartz, O. S. Ollila, T. Nylander and D. Topgaard, J. Magn. Reson., 2013, 230, 165-175.

44 A. Ribeiro, N. G. Wade-Jardetzky, R. King and O. Jardetzky, Appl. Spectrosc., 1980, 34, 299-305. 
45 L. E. Kay, D. A. Torchia and A. Bax, Biochemistry, 1989, 28, 8972-8979.

46 V. R. F. Matias, A. Al-Amoudi, J. Dubochet and T. J. Beveridge, J. Bacteriol., 2003, 185, 6112-6118.

47 S. Domingo Köhler, A. Weber, S. P. Howard, W. Welte and M. Drescher, Protein Sci., 2010, 19, 625-630.

48 A. Sverzhinsky, J. W. Chung, J. C. Deme, L. Fabre, K. T. Levey, M. Plesa, D. M. Carter, P. Lypaczewski and J. W. Coulton, J. Bacteriol., 2015, 197, 1873-1885.

49 H. Celia, N. Noinaj, S. D. Zakharov, E. Bordignon, I. Botos, M. Santamaria, T. J. Barnard, W. A. Cramer, R. Lloubes and S. K. Buchanan, Nature, 2016, 538, 60-65.

50 P. E. Klebba, J. Bacteriol., 2016, 198, 1013-1021.

51 K. Yonekura, S. Maki-Yonekura, R. Matsuoka, Y. Yamashita, H. Shimizu, M. Tanaka and F. Iwabuki, eLife, 2018, 7, e37833.

52 R. S. Peacock, A. M. Weljie, S. P. Howard, F. D. Price and H. J. Vogel, J. Mol. Biol., 2005, 345, 1185-1197.

53 D. D. Shultis, M. D. Purdy, C. N. Banchs and M. C. Wiener, Science, 2006, 312, 1396-1399.

54 P. D. Pawelek, N. Croteau, C. Ng-Thow-Hing, C. M. Khursigara, N. Moiseeva, M. Allaire and J. W. Coulton, Science, 2006, 312, 1399-1402.

55 S. J. Hickman, R. E. M. Cooper, L. Bellucci, E. Paci and D. J. Brockwell, Nat. Commun., 2017, 8, 14804.

56 J. L. Sarver, M. Zhang, L. Liu, D. Nyenhuis and D. S. Cafiso, Biochemistry, 2018, 57, 1045-1053.

57 J. S. Oeemig, O. S. Ollila and H. Iwaï, PeerJ, 2018, 6, e5412. 58 J. Evans, B. Levine, I. Trayer, C. Dorman and C. Higgins, FEBS Lett., 1986, 208, 211-216.

59 S. Brewer, M. Tolley, I. Trayer, G. Barr, C. Dorman, K. Hannavy, C. Higgins, J. Evans, B. Levine and M. Wormald, J. Mol. Biol., 1990, 216, 883-895.

60 D. M. Freed, S. M. Lukasik, A. Sikora, A. Mokdad and D. S. Cafiso, Biochemistry, 2013, 52, 2638-2648.

61 M. Krzeminski, J. A. Marsh, C. Neale, W.-Y. Choy and J. D. Forman-Kay, Bioinformatics, 2012, 29, 398-399.

62 C. J. Dickson, B. D. Madej, A. A. Skjevik, R. M. Betz, K. Teigen, I. R. Gould and R. C. Walker, J. Chem. Theory Comput., 2014, 10, 865-879.

63 J. Melcr, H. Martinez-Seara, R. Nencini, J. Kolafa, P. Jungwirth and O. H. S. Ollila, J. Phys. Chem. B, 2018, 122, 4546-4557.

64 M. R. Jensen, L. Salmon, G. Nodet and M. Blackledge, J. Am. Chem. Soc., 2010, 132, 1270-1272.

65 W. L. Jorgensen, J. Chandrasekhar, J. D. Madura, R. W. Impey and M. L. Klein, J. Chem. Phys., 1983, 79, 926-935.

66 J. L. F. Abascal and C. Vega, J. Chem. Phys., 2005, 123, 234505.
67 M. J. Abraham, T. Murtola, R. Schulz, S. Páll, J. C. Smith, B. Hess and E. Lindahl, SoftwareX, 2015, 1-2, 19-25.

68 O. H. S. Ollila, HpTonB(30-285) simulation Amber ff99SB-ILDN, 2020, DOI: $10.5281 /$ zenodo.3754109.

69 O. H. S. Ollila, HpTonB(30-285) simulation CHARMM36m, 2020, DOI: $10.5281 /$ zenodo.3743358.

70 O. H. S. Ollila, HpTonB(30-285) simulation Amber ffo3ws, 2020, DOI: 10.5281 /zenodo.3754180.

71 M. Kohagen, P. E. Mason and P. Jungwirth, J. Phys. Chem. B, 2016, 120, 1454-1460.

72 E. Pluhařová, H. E. Fischer, P. E. Mason and P. Jungwirth, Mol. Phys., 2014, 112, 1230-1240.

73 I. S. Virtanen and O. H. S. Ollila, $M D$ simulation of HpTonB(30-285) in $40 \mathrm{mM} \mathrm{NaCl}$ with Amber ffo3ws force field, 2020, DOI: 10.5281/zenodo.3756664.

74 I. S. Virtanen and O. H. S. Ollila, $M D$ simulation of HpTonB(30-285) in $150 \mathrm{mM} \mathrm{NaCl}$ with Amber ffo3ws force field, 2020, DOI: 10.5281/zenodo.3756668.

75 O. H. S. Ollila, HpTonB(179-285) simulation Amber ff99SB-ILDN, 2020, DOI: 10.5281/zenodo.3754253.

76 O. H. S. Ollila, HpTonB(179-285) simulation Amber ffo3ws, 2020, DOI: 10.5281 /zenodo.3754230.

77 O. H. S. Ollila, HpTonB(194-285) simulation Amber ffo3ws, 2020, DOI: 10.5281/zenodo.3754272.

78 L. Carlier, S. Balayssac, F.-X. Cantrelle, L. Khemtémourian, G. Chassaing, A. Joliot and O. Lequin, Biophys. J., 2013, 105, 667-678.

79 M. A. Kiirikki and O. H. S. Ollila, EN2(143-259) simulation with Amber ffo3ws, 2020, DOI: 10.5281/zenodo.3778216.

80 M. A. Kiirikki and O. H. S. Ollila, Mutated EN2(143-259) simulation with Amber ffo3ws, 2020, DOI: 10.5281/zenodo. 3778112.

81 O. H. S. Ollila, ProteinDynamics, 2018, DOI: 10.5281/zenodo. 1288574 .

82 S.-H. Bae, H. J. Dyson and P. E. Wright, J. Am. Chem. Soc., 2009, 131, 6814-6821.

83 C.-Y. Lu and D. A. V. Bout, J. Chem. Phys., 2006, 125, 124701.

84 G. Barbato, M. Ikura, L. E. Kay, R. W. Pastor and A. Bax, Biochemistry, 1992, 31, 5269-5278.

85 N. A. Farrow, R. Muhandiram, A. U. Singer, S. M. Pascal, C. M. Kay, G. Gish, S. E. Shoelson, T. Pawson, J. D. FormanKay and L. E. Kay, Biochemistry, 1994, 33, 5984-6003.

86 W. F. Vranken, W. Boucher, T. J. Stevens, R. H. Fogh, A. Pajon, M. Llinas, E. L. Ulrich, J. L. Markley, J. Ionides and E. D. Laue, Proteins, 2005, 59, 687-696.

87 O. H. S. Ollila, Data related to manuscript titled "Heterogeneous dynamics in partially disordered proteins", 2020, DOI: 10.5281/zenodo.3975900. 